\title{
Design requirements for effective hybrid decision making with Evolvable Assembly Systems
}

\author{
David Golightly, David Sanderson, Paul Holmes, Svetan Ratchev, Sarah Sharples \\ Faculty of Engineering \\ University of Nottingham \\ Nottingham, UK \\ \{david.golightly,david.sanderson,p.holmes,svetan.rachev, sarah.sharples\}@nottingham.ac.uk
}

\begin{abstract}
This paper examines 10 challenges for making automation a team player (Klein et al., 2004) in the context of Evolvable Assembly Systems (EAS) with the aim of delivering requirements for effective hybrid human-automation decision making. Specific decision making use cases for a demonstrator system were analysed to capture opportunities and requirements for effective human-agent cooperative decision making. These requirements covered agent design, human-machine interface design, context aware computing requirements and human competency. As such, the paper provides concrete examples of how general principles for hybrid decision making can be applied to EAS, and presents a pilot of a method for future requirements elicitation.
\end{abstract}

\section{Author Keywords}

Human-machine interface requirements; human-agent collaboration; human factors for manufacturing.

\section{INTRODUCTION}

While the manufacturing industry has historically used computers and automation to improve throughput, profitability, and quality, it is only recently that it has begun to fully take advantage of the flexibility, resilience and monitoring offered by intelligent control. This new domain of dynamic, networked, decentralised, and adaptive digital systems is often termed "Advanced Manufacturing". Advanced manufacturing systems are able to evolve and adapt to changes in product requirements in order to meet demand for those products. Assembly automation is a key step towards improved profitability and competitiveness in high labour cost areas such as the UK, and responsiveness and customisability are central to the future of manufacturing [1].

There is a lack of approaches addressing the industrial

Paste the appropriate copyright/license statement here. ACM now supports three different publication options:

- ACM copyright: ACM holds the copyright on the work. This is the historical approach.

- License: The author(s) retain copyright, but ACM receives an exclusive publication license.

- Open Access: The author(s) wish to pay for the work to be open access. The additional fee must be paid to ACM.

This text field is large enough to hold the appropriate release statement assuming it is single-spaced in Times New Roman 8-point font. Please do not change or modify the size of this text box.

Each submission will be assigned a DOI string to be included here. requirements of rapid response to constantly changing product requirements, and providing context-aware, online reconfiguration of the production line. The Evolvable Assembly Systems (EAS) project is aimed at the challenges posed by the production of highly-customisable products and specifically focusing on product-process-system coevolution. An EAS is characterised by being autonomous, context-aware and able to co-evolve with products, processes, and the business and social environment. Automation and machine intelligence is critical to this vision. An EAS may, for example, use a decentralised intelligence to connect multiple robotic arms and achieve highly adaptable and reconfigurable assembly of a variety of products. Human/machine collaboration and uncertaintyaware fixturing are also key enablers for the adaptive production of a product range.

Historically, attempts to integrate automation in many domains have taken a functional allocation approach [2]. One or more functional aspects of a process are identified as amenable to automation, and this aspect of the process is taken out of the control of the human and placed in the hands of some automated system. While this approach appears pragmatic, it can have significant negative repercussions for other tasks that remain within the remit of the human operator, for the maintenance of human skill, knowledge and motivation, and for the ability of humans to re-establish effective control if the automation fails $[3,4]$.

These negative effects are felt most acutely when the automation is taking over a cognitive aspect of control, such as decision-making or planning. These effects include both

1) decreased situation awareness for the operator who, without active involvement in the process, feels 'out of the loop' in both the status of the process, and the actions of the automation

2) the automation only considering a subset of the tacit and contextual cues, or secondary planning considerations, that a human operator brings to the problem. As a result, the functionally limited scope of the automation leads to suboptimal solutions that a human operator must repair. In the most extreme cases the operator may choose to turn off the automation altogether [4].

Hollnagel and Woods [5] propose an alternative approach to function allocation. They propose that humans, agents 
and artefacts should be viewed as a single system working towards control of a given domain - a 'Joint Cognitive System'. Based on cybernetics, they argue that control is a cyclical process and together automation and humans shape / regulate the performance of a process within required parameters. To optimise the Joint Cognitive System, the aim should not be to allocate function but to

1. Understand the goals and performance criteria required for effective control of the process.

2. Understand how control is achieved, irrespective of the actor or agent.

3. Design an approach for joint control of the domain.

Through these steps it will be possible to determine knowledge needs of human and automated agents, and the requisite overlap between the two so that (1) the operator maintains awareness of the right aspects of the process and the automation (2) the autonomous agent(s) has access to the right tacit, contextual and secondary planning considerations. Importantly, neither humans nor agents need complete visibility of each other's intentions, knowledge or plans. They simply need to know enough for mutual coordination, given the control requirements of a system. Certainly, however, knowing less than required for mutual coordination will lead to suboptimal decisions.

Klein et al. [6] argue that collaboration between human actors and agents should be based around more fundamental principles of collaboration. These requirements are

1. The basic compact - This is the agreement, often tacit, between parties to work towards a common goal. This agreement also entails the understanding that this is a process that needs investment and ongoing maintenance to ensure that goals remain current, mutual and shared. Critically, parties may need to explicitly indicate when they are temporarily or permanently suspending their involvement in the compact. For EAS, this is the agreed compact to work together to produce assembled goods to certain levels of quantity, quality etc.

2. Mutual predictability - Any party involved in a collaborative activity must be able to predict and influence the actions of others in the compact. In the Evolvable Assembly setting, this is the agreement that any party, including agents, is empowered to adapt assembly parameters within agreed, predictable limits.

3. Directability - A party must also be able to shape another party's activity and requires the other party's adequate responsiveness. In the Evolvable Assembly setting, this is the ability not only for the operator to shape the activity of agents, but also for the agents to make requests of the operator to help fulfil the activities of the Evolvable Assembly Cell (e.g. to assist in swapping two robots when one fails).
4. Common ground - Common ground is shared beliefs, knowledge and awareness requisite to complete a task. As important as the common ground itself is the need for all parties to work to maintaining that common ground by sharing information about overall task status, and their own status, or by acknowledging when a party's knowledge may be incomplete. In EAS, this is the ability on the part of all actors to update all other actors on their availability to perform assembly tasks, to understand shared goals of the cell, and to understand (and be sensitive to) the capabilities and limits of other humans, agents, autonomous robots within the cell etc.

From these four principles for collaboration, Klein et al. argue that there are ten challenges to address when one or more of the team players in a collaborative control setting is an automated agent. These challenges are particularly acute when intelligence (i.e. decision making) might be distributed between human and non-human actors. Ten challenges are presented in Table 1, along with some examples of relevance for EAS.

One of the conclusions of Klein et al. is that not all of these challenges can be addressed by the design and operation of the agents. If we take Challenge $1-$ taking part in the compact, this proposes that an agent needs to give information about its status, and have an understanding of its goals and role in a joint activity. Having automation give information about the availability of itself and the process its controls should be achievable with current technology; having an agent 'understand' other goals and intentions is less feasible at this time, and poses a more general challenge for future automation design.

Therefore, one task in the design of an EAS is to understand how to utilise other mechanisms that may be available to support human-agent coordination. This might be in terms of shifting the cognitive burden from the agents to the humans (i.e. the human needs greater awareness of the state of the system to compensate for limited capabilities of agents), though this is not often desirable because of the workload involved (see Challenge 10). A more useful approach is to think of other technical means to give the system the information it needs for coordination. An example of this is context-sensitivity [7]. Rather than requiring the agents to intelligently determine the status and availability of an operator, or requiring the operator to manually inform the agents of their availability, context awareness (e.g. through location and task sensing) could calculate availability on the behalf of the operator and send that as a parameter for the agents to take into account.

The work reported in the rest of this paper took the challenges and interpreted them with specific reference to a candidate EAS, in order to consider where the burden of addressing the challenges may lie:-

- Through agent design - ensuring the agent has adequate models and algorithms for collaboration. 
Challenge 1: To be a team player, the agent must take part in the basic compact Automation must be able to say when it is able to engage in mutual activity. The human operator must be aware of when automation, or the processes it controls, is no longer available to support the goals.

Challenge 2: Agents must model other actors' intentions In the counterpart to Challenge 1, agents must also be able to understand the availability and intentions of other actors within the system.

Challenge 3: Humans and agents must be mutually predictable The actions of agents must be predictable to human operators. This is a challenge when decision-making or action is emergent from a number of other processes or when the algorithm is opaque to humans (Balfe et al., 2012)

Challenge 4: Directability Agents must be governed or directed in some manner, and having the means to do so effectively is critical to the success of human-automation collaboration.

Challenge 5: Making status and intentions obvious One of the key difficulties typically encountered with automation is that the operator does not have an understanding of the plans or actions of the automation. Therefore, agents must make their intentions clear in a manner that matches the control responsibilities of the operator (Hollnagel and Woods, 2005).

Challenge 6: Agents must be able to observe and interpret other actors' intentions Agents must be able to infer from signals the implications of other actors' actions for overall collaborative control. In this sense, the agent has to, to a degree, model other actors.

Challenge 7: Agents must engage in goal negotiation In order to maintain the compact, agents must communicate their goals and leave them amenable to adaptation by human users. Likewise they must be able to express their goals and communicate their priority should the human actor need to adapt their own goals in response.

Challenge 8: Technologies for planning and autonomy must take a collaborative approach Plans are typically iterative and subject to change. Agents developing and executing plans must be open to reallocation and renegotiation of resources in response to changing environmental conditions.

Challenge 9: Agents must participate in managing attention Automated agents should not just highlight when they are at the edge of performance, but also indicate when performance barriers are being reached or when substantive system changes are about to take place.

Challenge 10: All team members must help control the cost of collaborative activity Coordination has a cost, and agents should have a model of workload in order to anticipate and manage demands placed on the operator. Likewise, the human operator should have a model of the capabilities and limits of the automation.
An autonomous agent must communicate when it, or the assembly capabilities it controls (e.g. a PLC), is no longer available or is degraded in some manner. Humans and agents should share, to a degree, knowledge and prioritisation of KPIs.

A human operator should also communicate when he or she is no longer available, for example if they are maintaining a different cell in another part of a facility. Agents should be designed to have a model of this availability.

There must be a clear behavioural model of the agents, and the EAS as a whole, available to the operator to interpret and predict events such as changes to scheduling to optimise production.

Agents in the EAS must be open to control from the human operator. In keeping with the principles of JCS, this means and level of control must be appropriate to the responsibilities of the human - for example, making decisions around the need for maintenance or load balancing across a number of EAS cells.

For EAS, this means making the actions of the agents salient to the operator through appropriate HMI design. Again, status and intentions must be represented at a level that is appropriate to the responsibilities of the human.

Agents in an EAS must have a model of other actors' actions and intentions. This could be other agents in the cell, such as robots within the cell environment, or with human operators. A sensored environment would help agents to interpret the position and actions of a human operator, for example are they currently involved in another task somewhere in the cell.

In the EAS context, goal negotiation is most usually around the scheduling, and re-scheduling, of assembly orders. Not only should goals be negotiable, they should be represented in the HMI in a manner that accords with the operator's key responsibilities.

Extending on Challenge 7, planning and re-planning must be collaborative. In EAS this is likely to mean the adaptation of production schedules based on new information or requirements. Therefore agents must be amenable to change. Likewise, agents that propose changes to a cell (e.g. to topology) must communicate the reasons for change in a meaningful manner.

In EAS, this may be relevant when tools have been replaced by new tools with new capabilities (e.g. a faulty robot is replaced with a lower specification robot), or when a topology has different performance characteristics. While the change may at first be positive, as time goes on, different orders may push that configuration to the limit.

In EAS, agents must be aware of the tasks and availability of a human operator, in order to manage workload. For example, a tool that an agent controls may need attention - the agent should be able to sense that a human operator may be already occupied, and adapt its work accordingly until the operator is available. Likewise, an operator needs a model of the capabilities of the agents, and the EAS as a whole. 
- Through Human Machine Interface (HMI) - both to give the human operator awareness of events, or to allow the human operator to inform the agents of change of status or plans, to accept or veto decisions etc.

- Through context aware computing - Context awareness and ubiquitous computing of the workstation, product or operator.

- Through human knowledge or procedures - Knowledge of policies and priorities relevant to the use of the EAS; training and support to ensure the operator has an adequate model of the EAS and agent capabilities.

\section{METHOD}

Having established some principles for a hybrid (JCS) approach to decision making with evolvable assembly, the next stage is to specify requirements for design and deployment. The nature of decision making, being highly contextual, requires that actual decision making use cases are used as the basis of analysis. Therefore, a target EAS implementation, the SMART demonstrator [8], has been used as a basis. The method applied is as follows

A description of the demonstrator was reviewed. The review of the demonstrator description was complemented by a tour of the demonstrator which was video recorded and transcribed as notes. From this description, a set of decisions were identified that were critical to the operation of the demonstrator. Three high level decisions, termed 'decision-making use cases', were identified. These three were selected on the basis that they represented key capabilities for an EAS - error recovery, flexible operation, and the ability to reconfigure resources.

For each decision making use case, the overall aims of the decision were discussed and understood between the analyst and a subject matter expert. This decision was then broken down into a number of decision steps. Rasmussen's decision ladder [9] was used as a guide to check that major aspects of the decision making process were addressed. Each decision step was then reviewed as to how it was currently performed. This gave a baseline for current operation of the demonstrator. These decision steps were then reviewed generally against the ten challenges, to understand where opportunities might lie for improving or enhancing hybrid decision making within the context of these challenges. Each decision step was then reviewed, in turn, as to how it could be supported using one of the opportunities for implementing hybrid decision making design of the agents, design of the HMI, use of context sensitive computing, and knowledge, competency and policy for human agents. Notes were recorded in a Microsoft Excel table.

\section{DEMONSTRATOR DESCRIPTION}

First, the technical analysis of the demonstrator was completed which highlighted a number of tasks, user roles, and performance characteristics. Relevant points for the decision making analysis are as follows.
The SMART SMC demonstrator comprises a small assembly cell - see Figure 1. This cell simulates an assembly task by presenting containers to an assembly line to which red, yellow or blue particulate may be added based on a given order. Each product is then tested by a potentiometer, and, if it meets the required quality standard, is packaged. Several products may be present on the assembly line at any one time. Workstations on the cell include (1) Container loading (2) Red particulate (3) Blue particulate (4) Yellow particulate (5) Testing station 1 (6) Testing station 2 (7) Lidding and labelling (8) Dispatch.

Orders are submitted by an interface where a human operator specifies the combination of particulate required. Without agents, each workstation in the cell is controlled by a Programmable Logic Controller (PLC) that provides basic automation control of the physical workstation (for example, one PLC controls the movement of the container within the 'yellow' filling station and controls whether, and how much, yellow particulate is added according to the customer order).

The intelligence of the cell comes from agents (implemented on 'Raspberry Pi's) that control each PLC associated with a given workstation. The agents are able to communicate to each other, including to a designated Point of Contact Agent (POCA) that interfaces with a VDU-based HMI that the operator uses to submit orders. Each agent is able also to control the PLC, for example to let a container pass through a workstation if no particulate is required, to let a container recirculate if a given stage of the assembly process cannot be completed at the right time, or to choose which of two testing available stations is to be used to measure the quality of the product. Thus, by communicating with each other and through the POCA, the agents together are able to adapt the order in which products are assembled to meet predefined criteria. In this manner they are able to demonstrate flexible, adaptive assembly, and provide product customisation down to a 'batch of one'. The role of the human operator with the demonstrator includes

- submitting orders to the cell via the POCA, as well as keeping consumables restocked.

- looking for error states and determining when an error state actually needs intervention and acting accordingly

- collecting completed product.

- potentially, to alter the configuration of the cell to meet differing product demands.

- turning on and off, cleaning and maintenance.

In theory, depending on workload, a front-line operator may have responsibility for multiple cells. One implication of this is the operator may not always be in physical proximity and will be remotely receiving information on the workstation. The assumption is that this information is accessed in real-time. 


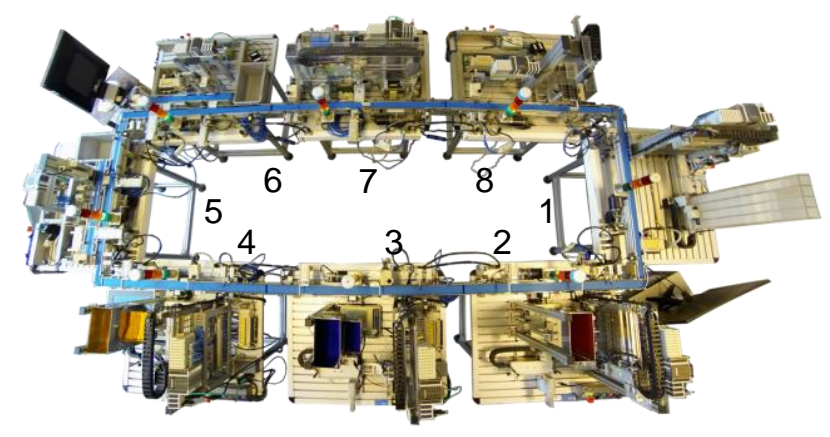

Figure 1 - SMART Demonstrator

\section{ANALYSIS}

From this description, eight potential decision making use cases (DMUCs) were identified. Three were used for further analysis as they captured the flexible nature of Evolvable Assembly Systems, and were cases where collaboration between human and agents is crucial.

DMUC1: Do I need to intervene to rectify a fault?

DMUC2: Following the removal of a part of the cell, can we continue to fulfil orders, potentially in a "degraded mode"?

DMUC3: What is the best topology for the cell (to improve performance)?

Several decision steps were identified for each decision. As noted in the method, the decision ladder was used as a prompt to what sub-decisions comprised the different phases of the decision making use case. Table 2 presents an example of the full analysis for DMUC1

\section{Requirements for Evolvable Assembly Systems}

In total, 13 unique requirements were identified for agent design; 20 requirements for HMI; 8 requirements for context sensitivity; 9 requirements for operator knowledge and policy. The implications of these requirements for the SMART demonstrator, and more widely for Evolvable Assembly Systems, are considered below

\section{Implications for Agent Design}

Three main implications arise from the requirements covered above. The first is an increased context awareness and integration with "ubiquitous sensing" equipment. The remaining two of these are in terms of the technical capabilities of the agents: there is a need for learning, and also for distributed planning.

Greater context awareness would not only involve better linkage with the PLCs in order to extract a greater amount of more finely-grained local state information, but also external information from other agents, the operator, and the equipment described elsewhere in the "context sensitivity" section. This would allow the agents to base their reasoning on a more accurate picture of the world around them, and identify the most appropriate action to take (Challenge 6 - Interpreting Other Actors). Agents that are able to learn from experience over time allow the system to fully utilise the information they are receiving from the rest of the system. Techniques such as fault prediction, optimisation of routing and topology, and suggesting more appropriate solutions based on past experience all become possible. Critically, the outputs of this learning must be predictable and visible to the operator (Challenge 3 - Mutual predictability).

The final implication is allowing the agents to leverage both their increased context-awareness and learning in a distributed planning approach. Although the agents currently distribute some of their decision-making, the inclusion of external information - as well as taking the operator into direct account - will require a more nuanced approach to this planning activity. This will allow the agents to take a more active, and effective, role in the tactical aspects of decision making, which can be proposed for negotiation (Challenge 7 - Goal Negotiation).

\section{Implications for $\mathrm{HMI}$}

The requirements highlight a number of needs for a main HMI that acts as a primary communication channel between the operator and agents. These requirements go beyond the current role of the HMI, which is to submit orders. Importantly the requirements identify this as a two way communication

- Output from the agents about current and future status, options that are available, or proposed changes (e.g. to topology or scheduling) (Challenge 5 - making status obvious).

- Input from the operator regarding their status, changes to the priorities of performance characteristics (e.g. to prioritise certain orders), or to confirm or veto agent options (Challenge 4 - Directability).

By making the assumption that agent-based control will be robust and error free, then operators will be free to control at a higher, more strategic level, relying on the agents to make successful implementation decisions. For the demonstrator, this constitutes decisions about whether proposed topology changes or replanning of the production scheduling are appropriate (Challenge 7 - Goal Renegotiation; Challenge 8 - Collaborative renegotiation). It is vital, however, that control is expressed on a KPI-type basis - that is, how will choices or decisions effect the overall performance of the workstation based on performance needs of the organization.

The HMI also offers opportunities to provide diagnostic information, guidance on how to maintain and operate the workstation, or how to install new components. This may be vital if the agents are presenting options or reconfigurations that are rarely encountered. It is acknowledged however, that not all diagnostic information or assembly guidance can be always accurate, and, in this regard, there is a requirement for the HMI to also accept 
DMUC 2. Following the removal of a part of the cell, can we continue to fulfil orders, potentially in a "degraded mode"?

\begin{tabular}{|c|c|c|c|c|c|}
\hline Decision Step & Current & Agents & HMI & Context Sensitivity & Human Operator \\
\hline $\begin{array}{l}2.1 \text { What capabilities } \\
\text { have been lost from } \\
\text { the cell? }\end{array}$ & $\begin{array}{l}2.1 \text { Agent can } \\
\text { communicate that it is no } \\
\text { longer available; operator } \\
\text { will know. }\end{array}$ & 2.1 N/A. & $\begin{array}{l}2.1 \text { Could be more } \\
\text { explanatory, rather than } \\
\text { informing by default, or the } \\
\text { current availability of } \\
\text { capabilities. }\end{array}$ & $2.1 \mathrm{~N} / \mathrm{A}$. & $\begin{array}{l}2.1 \text { What capabilities are } \\
\text { related to which physical } \\
\text { resources. }\end{array}$ \\
\hline $\begin{array}{l}2.2 \text { Do alternatives } \\
\text { exist and if so what } \\
\text { are they? }\end{array}$ & $\begin{array}{l}2.2 \text { Agent knows if there } \\
\text { is a duplicate; operator } \\
\text { should know. }\end{array}$ & 2.2 N/A. & $\begin{array}{l}2.2 \text { Could offer alternatives } \\
\text { via HMI. }\end{array}$ & $\begin{array}{l}2.2 \text { Location of alternatives, } \\
\text { current workload and } \\
\text { useage. }\end{array}$ & $\begin{array}{l}2.2 \text { Knowledge of } \\
\text { alternatives and capabilities } \\
\text { / limitations; Operator } \\
\text { having veto / confirmation } \\
\text { of use of an alternative; }\end{array}$ \\
\hline $\begin{array}{l}2.3 \text { Are there } \\
\text { alternatives with } \\
\text { adaptation? }\end{array}$ & $\begin{array}{l}2.3 \text { Agents wouldn't know } \\
\text { but the human could } \\
\text { identify resources suitable } \\
\text { for adaptation. }\end{array}$ & $\begin{array}{l}2.3 \text { Agents would be able } \\
\text { to identify suitable } \\
\text { candidates for adaptation } \\
\text { and perform themselves } \\
\text { or request assistance; } \\
\text { understand implications } \\
\text { of new capability. }\end{array}$ & $\begin{array}{l}2.3 \text { Could inform the } \\
\text { operator of agents' } \\
\text { recommendations for } \\
\text { adaptations, or inform of the } \\
\text { nature of carried out } \\
\text { adaptation and implications } \\
\text { for capability. }\end{array}$ & $\begin{array}{l}2.3 \text { Location of alternatives, } \\
\text { current workload and } \\
\text { useage. }\end{array}$ & 2.3 Same as 2.2. \\
\hline $\begin{array}{l}2.4 \text { If more than one } \\
\text { exists which one do } \\
\text { you choose? }\end{array}$ & $\begin{array}{l}\text { 2.4 Agent makes decision } \\
\text { but not visible to operator; } \\
\text { operator has no say in it. }\end{array}$ & $\begin{array}{l}2.4 \text { Agent to make choice } \\
\text { on alternatives, give } \\
\text { options to operator based } \\
\text { on capabilities and } \\
\text { predictions against KPIs. }\end{array}$ & $\begin{array}{l}\text { 2.4 Present alternatives and } \\
\text { result of choice with } \\
\text { appropriate veto / } \\
\text { confirmation - KPI-based. }\end{array}$ & $\begin{array}{l}\text { 2.4 Current state external to } \\
\text { the cell e.g. are other cells } \\
\text { available. }\end{array}$ & $\begin{array}{l}2.4 \text { Agree or veto agents' } \\
\text { decision, or make a decision } \\
\text { on their own. }\end{array}$ \\
\hline $\begin{array}{l}2.5 \text { Can the schedule } \\
\text { be adapted based on } \\
\text { the available } \\
\text { capability? }\end{array}$ & $\begin{array}{l}2.5 \text { Agent does not know; } \\
\text { human has to work it out. }\end{array}$ & $\begin{array}{l}2.5 \text { Agent has knowledge } \\
\text { of current schedule, and } \\
\text { reschedule based on } \\
\text { adjusted capability. }\end{array}$ & $\begin{array}{l}2.5 \text { Presents new schedule } \\
\text { with opportunity to veto; } \\
\text { Opportunity for operator to } \\
\text { prioritise KPIs to shape } \\
\text { rescheduling. }\end{array}$ & $\begin{array}{l}2.5 \text { Might give operator } \\
\text { notice on availability of } \\
\text { fully functioning } \\
\text { alternatives, that could } \\
\text { influence reschedule. }\end{array}$ & $\begin{array}{l}2.5 \text { Ability to prioritise } \\
\text { KPIs, accept / veto plan. }\end{array}$ \\
\hline $\begin{array}{l}\text { 2.6 Which orders still } \\
\text { in the cell can you } \\
\text { finish? }\end{array}$ & $\begin{array}{l}2.6 \text { Agents know that } \\
\text { orders can't be fulfilled } \\
\text { but can't act on it; } \\
\text { operator has to be aware } \\
\text { of it. }\end{array}$ & $\begin{array}{l}2.6 \text { Agent is aware of } \\
\text { alternative possibilities } \\
\text { for incomplete orders. }\end{array}$ & $\begin{array}{l}2.6 \mathrm{HMI} \text { communicates } \\
\text { number / location of orders } \\
\text { that cannot be completed. }\end{array}$ & $\begin{array}{l}2.6 \text { Product sensing to flag } \\
\text { is not able to complete. }\end{array}$ & 2.6 N/A. \\
\hline
\end{tabular}

Table 2 - Example output for Decision Making Use Case 2 
notes or clarifying details from the operator in the form of knowledge management input. By having more regular, standardised processes for physical activity, the tasks of the operator become more predictable (Challenge 3 - Mutual predictability).

The requirements also highlighted the value of a wearable or portable device. One of the limitations of the current demonstrator assembly environment is the need for the operator to visually survey the cell to monitor if there is a problem with assembly, for example through identifying when 'traffic light' indicators on a workstation indicate a problem. Not only does this put the cognitive burden of understanding workstation availability onto the operator, this limits the ability of the operator to carry out work away from the cell. Haptic and visual feedback on a wearable / portable device can allow the agents to alert the operator to current and potential future availability issues, wherever the operator is located (Challenge 9 - managing attention). The wearable HMI can also suggest routes in situations where the operator needs to find equipment (DMUC 2), or is working on multiple or geographically dispersed cells and workstations, for example when working with the assembly of large components (e.g. aircraft wings).

\section{Context sensitivity}

This requirements elicitation exercise has identified specific applications and outputs of context sensitive computing. First, sensing of the products / assemblies themselves can be applied to understanding product status. This gives greater diagnostic power to the agents in understanding when and how there may be problems with the assembly of a product maintained. This information can be conveyed to the operator, giving greater awareness of the availability of different parts of the workstation to fulfill the basic compact (Challenge 1 - maintaining the compact). Second, there is context (location) sensing of other alternatives to current workstations or capabilities. While this is trivial for the current demonstrator, it is possible that a full EAS, for example using interchangeable robots, will benefit from knowledge of status and location of alternatives when making decisions about replacements (DMUC2) or changes to topology (DMUC3). Third, there is knowledge of the operator both in terms of location and current task, which might be inferred from location. This operator sensing is crucial as it would allow the agents to have a rudimentary model of the availability of the operator, which then can be factored in when considering the predictability of the operator to assist in an activity (Challenge 6 - interpret other actors intentions), or operator workload (Challenge 10 - controlling the cost of collaboration).

\section{Human capability}

The review of the current status of the demonstrator highlighted a high reliance on the human operator to maintain situation awareness of the assembly line, to diagnose faults, and to interpret changes to the topology (the ordering of tasks as they are performed by the workstation). This kind of heavy reliance on operator skill and knowledge is by no means unrepresentative of many legacy assembly line environments currently working without agent-based capability.

The analysis performed here offers some concrete paths forward. It allows us to see that there are two key competencies for the operator of the cell. The first is in targeting (i.e. strategic planning) (Hollnagel and Woods, 2005). If the future responsibility of the operator is to understand and contribute to decisions regarding the overall performance of the manufacturing cell, then the onus should be less on 'sensing' the performance on the cell as this can be done much more effectively by the agents combined with context sensitivity. Instead, the operator is best placed to interpret the mapping between cell performance and the KPIs (Challenge 7 - Goal Negotiation). Therefore, knowledge for the operator will be based on knowing and interpreting the mapping between KPIs and the capabilities of the cell, and training and knowledge should be focussed in that direction. Crucially, however, this must be in order to maximise (and not be a replacement for) the quality of information the operator receives via the HMI. The second role of the operator is going to be one of a physical assistant, for example during physical reconfiguration of the assembly line, or restocking supplies. To this end, the operator can be supported through the HMI through the availability of diagnostic or installation procedures, but knowledge, training and skill development must still be focussed on this aspect of their competency.

\section{Methodology}

The methodology proved useful in highlighting a set of requirements across different aspects of the evolvable assembly environment. This has allowed us to turn general ambitions, such as the use of context awareness (Suh et al., 2008), into specific needs to support specific activities. As this was a pilot of a method, a number of observations about how to use or improve the method came to light.

- The identification of the candidate set of decision making use cases could be made more robust and exhaustive in the future, by using a structured elicitation approach such as Hierarchical Task Analysis. It is envisaged that a comprehensive high-level task breakdown could be then used to identify key decisions for each phase of work.

- Not all decisions followed a linear pattern. This was particularly the case for DMUC2 where there were a number of avenues that could be explored depending on whether an exact replacement was available, whether a replacement with different or reduced capabilities was available, or whether no replacement was available at all. In future, it might be useful to use a flow diagram to structure the elicitation of the decision making stages. 
- It became apparent that many decision branches were shared across decision making use cases. For example, many of the steps associated with making decisions on alternative arrangements of the cell applied to both DMUC2 and DMUC3. Therefore, there is likely to be a rapid tail-off in the number of decision making use cases, and stages, required to capture the majority of decision-based requirements.

- The approach used here involved primarily one analyst and one subject matter expert. However, it would appear feasible to use the method in a group setting. This is likely to elicit more requirements and enable validation.

\section{Limitations}

As well as the points about the method raised above, there were several other limitations of the study. First, while the use of the ten challenges (and the four underpinning principles of cooperation) have informed this study, they have not been used in an explicitly structured manner, for example as some kind of checklist. The danger with such an approach is that it would make the method too unwieldy, but caution should be noted that without being vigilant to reflect on the challenges at multiple points during the elicitation, it is easy to forget their relevance. Second, the method has worked well for a limited decision set on a small scale problem. While the method shows promise, it needs to be verified as to whether it will scale for a substantially more complex EAS.

\section{CONCLUSIONS AND NEXT STEPS}

The approach presented in this paper has shown how an alternative to function allocation can be used with Evolvable Assembly Systems to arrive at requirements for hybrid decision making. These requirements, based in Klein et al.'s challenges for automation as a team player, can help to alleviate problems of brittleness or inflexibility in agentbased assembly. Key findings include

- An instantiation of ten challenges for EAS. This can be used as a guide in future EAS developments

- Examples of the application of general technologies, now tied to specific decision making use cases

- A methodology that can be applied to other decision making scenarios for EAS

- Decision-centred applications of technologies including wearable HMI and context sensitivity to provide information about operator intention and availability that gives the agents a more predictable model of operator behavior

- Emphasis on the importance of presenting the operator with interfaces that present and receive input at a monitoring or targeting level - this is likely to involve interfaces that reflect KPIs in some manner

Next steps to develop hybrid decision making for EAS comprise
1) Repeat the process for remaining decisions for the SMART demonstrator.

2) Move from requirements to implementation specification. For example, having identified the need for KPI-based control, the next stage of work can look at approaches such as Ecological Interface Design [10] to arrive at specific HMIs.

3) Empirically evaluating the impact of the requirements on the successful operation of the demonstrator (this could be compared with a baseline of human / agent performance under current operating conditions).

4) Repeat the process for an alternative form of EAS - for example, one with a greater degree of robotics.

By synthesizing the requirements from two or more EAS, it will be possible to move from specifying requirements for individual EAS implementations, to specifying more general patterns that apply to all types of EAS.

\section{ACKNOWLEDGMENTS}

The reported research has been funded by the EPSRC grant $\mathrm{EP} / \mathrm{K} 018205 / 1$, the support of which is gratefully acknowledged.

\section{REFERENCES}

[1] Rhodes, C. (2015). Manufacturing: Statistics and Policy. Briefing Paper. House of Commons Library.

[2] Fuld, R. B. (2000). The fiction of function allocation, revisited. International Journal of Human-Computer Studies, 52(2), 217-233.

[3] Bainbridge, L. (1983). Ironies of automation. Automatica, 19(6), 775-779.

[4] Balfe, N., Wilson, J. R., Sharples, S., \& Clarke, T. (2012). Development of design principles for automated systems in transport control. Ergonomics, 55(1), 37-54.

[5] Hollnagel, E., \& Woods, D. D. (2005). Joint cognitive systems: Foundations of cognitive systems engineering. CRC Press.

[6] Klein, G., Woods, D. D., Bradshaw, J. M., Hoffman, R. R., \& Feltovich, P. J. (2004). Ten challenges for making automation a "team player" in joint human agent activity. IEEE Intelligent Systems, (6), 91-95.

[7] Suh, S. H., Shin, S. J., Yoon, J. S., \& Um, J. M. (2008). UbiDM: A new paradigm for product design and manufacturing via ubiquitous computing technology. Int. J. of Computer Integrated Manufacturing, 21(5), 540-549.

[8] Chaplin, J. C., Bakker, O. J., de Silva, L., Sanderson, D., Kelly, E., Logan, B., \& Ratchev, S. M. (2015). Evolvable Assembly Systems: A Distributed Architecture for Intelligent Manufacturing. IFAC-PapersOnLine, 48(3), 2065-2070.

[9] Jenkins, D. P., Stanton, N. A., Salmon, P. M., Walker, G. H., \& Rafferty, L. (2010). Using the decision-ladder to add a formative element to naturalistic decision-making research. Intl. J, of Human-Computer Interaction, 26(2-3), 132-146.

[10] Vicente, K. J. (2002). Ecological interface design: Progress and challenges. Human Factors, 44(1), 62-78 